\title{
EL PRÓLOGO EN LA TRAGEDIA DE SÓFOCLES
}

\author{
(Continuación)
}

IU.-Sistematización: en busca de esquemas homogéneos.

Una vez que hemos ido analizando uno a uno todos los Prólogos de la obra de Sófocles, debemos dar un paso más tratando ahora de introducir en el material estudiado una ordenación que nos pusibilite obtener unos comportamientos regulares de nuestro autor, lo que en definitiva nos ponga de manifiesto su técnica dramática. Iremos aludiendo por separado a cada uno de los cuatro aspectos tiadicionales de nuestro tipo de análisis, y al final de cada uno trataremos de sacar las conclusiones parciales.

\section{A) Estructura formal del Prólogo en Sófocles.}

I) Tipologia del Prólogo seguin sus participantes.

Prescindiendo en un primer momento de la especielización en su relación con el coro, que en realidad aún no ha salido, podemos fijir dos tipos:

a) actor/actor: Ant., Fil. y Rastr.

b) actor/actor/actor: Ay., Traq., E. R., El. y E. C.

Por 1o tanto vemos que hay un uso preferente del esquema de tres actores, lo que habremos de tener presente para cuando en el punto siguiente tratemos de hacer la posición de Sófocles en la evolución del Teatro. Pero precisemos aún más, y observemos que el empleo de tres actores lo hace el poeta desde sus obras más antiguas, aunque en su etapa intermedia, teniendo en cuenta solamente las piezas conservadas, hay una inclinación al uso de dos actores: a partir de Antigona hasta Filoctetes, puesto que Electra es un caso en el que la intervención 
del tercer actor es muy reducida al final de la escena -recordemos que se trata del lamento de Electra misma desde dentro del palacio y consiste simplemente en un dímetro anapéstico-, y en estas circunstancias sólo el Edipo Rey hace excepción a ese período de nuestro poeta.

Ahora bien, también es importante tener en cuenta la realización concreta de cada uno de los mencionados actores. Ya hemos dicho anteriormente que ell el caso presente del Prólogo no es exacto esto de caracterizar a los participantes con los tipos antiguos, puesto que el coro aún no ha llegado a la orchestra, y por lo tanto todavía no están caracterizados en relación con los diferentes actores individuales, sin embargo, nosotros a la vista de toda la obra hemos preferido hacerlo para un estudio más preciso, ya que nuestra situación lógicamente no es la misma que la del auditorio que presenciaba la puesta en escena de la obra y que todavía en el Prólogo no podía establecer las diferentes relaciones de los actores con el coro. Pues bien, teniendo en cuenta ahora los diferentes tipos antiguos podemos introducir una nueva clasificación:

a) J.C./P.S.: Fil. y Rastr.

b) J.C./P.S./P'.S'.: Ay., Traq., E. R., El. y E. C.

c) Op./P.S.: Ant.

Frente a la actuación casi constante del J.C. vemos cómo se le pueden añadir un P.S. o dos. Ahora bien, si tenemos presente el tipo del mpó-

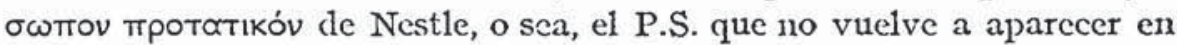
la marcha de la obra, conviene hacer notar cómo en Sófocles se da con una relativa frecuencia este empleo, puesto que lo encontramos en tres casos: Ayax, Edipo Rey y Edipo en Colono, aunque también debemos hacer constar que en las tres ocasiones se trata de escenas de tres actores, razón por la que el esquema antiguo se halla complicado, pero, eso sí, a partir de una situación antigua. De las cinco veces en que en-

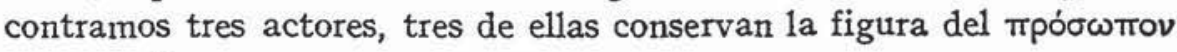
тротатікóv.

2) Tipología del Prólogo segín su estructura formal interna.

Para una exposición más detallada iremos introduciendo una serie de divisiones, como hemos hecho al analizar los ejemplos de forma individual, $\mathrm{y}$ así estableceremos cuatro partes:

I) Preparación.-Es el primer momento del Prólogo, en el cual se dispone la escena para dar paso a continuación al núcleo. Esste pe- 
ríodo inicial, sin embargo, podemos a su v' $\mathrm{c}$ fragmentarlo en dos partes:

a) Entrada.-Que expresa la 1legada del o de los diferentes actores. En Sófocles hay dos procedimientos formales para este momento

a) Esquema simple: $r$, en $A y$. y El. En estos ejemplos una resis de un actor enmarca la escena.

$\beta)$ Esquema complejo: puede suceder que se dé un esquema más complicado, en el que hay una mezcla de resis y elementos dialógicos, como:

$$
\begin{aligned}
& -r .+r .+d . e .3: \text { Ant. y } E . C . \\
& -r .+ \text { d.e.3 }+ \text { r.: Fil. }
\end{aligned}
$$

ejemplos éstos en los que el poeta quiere, por razones especiales de la obra, exponer de forma más amplia la llegada de los actores.

b) Narración previa.-En este primer periodo de preparación el poeta hace a veces referencias a una historia anterior y básica para el núcleo de la escena, que será la narración principal. Este es el caso de Traq. y E. R., cuyas estructuras formales son: $r .+r . y r .+r .+r .+$ d.e.3, respectivamente.

En definitiva, en este primer momento del Prólogo, y antes de que llegue el núcleo de la escena, podemos encontrarmos con una simple resis o bien con una estructura más compleja, en la que la duplicación de la resis por la presencia de más de un actor se puede unir a un elemento dialógico, es decir, un esquema: $r .+r .+d . e .3$, aunque lógicamente esto pucde alterarse de diferentes mancras, dando los diversos casos que hemos aludido. Por otro lado, este comportamiento a partir de un elemento simple es frecuente en nucstro autor en periodos ya meramente dialógicos de su obra, lo que nos confirma ya descle ahora lo que decíamos en el punto primero de este trabajo al adelantar el hecho de que el Prólogo, por ser una escena plcnamente perteneciente ya al ámbito del Teatro, es expresado mediante estructuras ya teatrales, por cuanto que no podía tener una forma previa, ya que no existía.

II) Nícleo.-Es el período en torno al cual gira el resto de la escena. En las obras conservadas tiene una estructura un tanto dispar, aunque sin embargo pueden observarse dos grupos generales:

a) Esquema arcaico:

- r.: en Ant.

- d.e. $3+$ r.: en Traq. y Fil.

- d.e.3 +r. +r.3: en E. C. 
En cualquiera de estas tres posibilidades encontramos un empleo antiguo, puesto que la resis podía ir desde, antiguo sola, o bien abierta, o cerrada, o ambas cosas a la vez, por un elemento dialógico. Ahora bien, esto no quiere decir que la regularidad de estos casos sea total, puesto que vemos que con una frecuencia superior se da la variante del d.c. en vez de la simple $e$. Por otro lado además, este elemento dialógico, aunque esto correspondería al aspecto del contenido y de la función, no tealiza simplenente la función de abrir o cerrar la resis, pero sobre estos pormenores ya hemos hablado al analizar cada ejemplo por separado, y remitimos allí; aquí sólo hacemos alusión para restringir el valor arcaico de estas estructuras.

b) Esquema innovado:

$$
\begin{aligned}
& \text { - d.e.3: E. R. } \\
& -r .+r .+e .3: \text { Rastr. } \\
& -r .+e .3+r .: \text { Ay. }
\end{aligned}
$$

Volvemos a encontrarnos con unos tipos de innovaciones formales frecuentes en nuestro autor. De las dos últimas acabamos de hablar en el apartado anterior de "preparación». Del d.e.3 también existen otros ejemplos en la obra de Sófocles, y tal vez supone un nivel ya importante de innovación, por cuanto que un elemento dialógico, que según los esquemas antiguos estaba subordinado a la resis, ahora ya ha obtenido tuna total independencia y funciona por sí solo.

Por lo tanto la parte del Prólogo en la que se expresa el núcleo de toda la escena, conserva en una cierta medida una estructura antigua, si prescindimos de los detalles innovadores. Pero también hay algunos casos que ya suponen un esquema nuevo, en los cuales podemos ver no obstante procedimientos ya empleados en otras escenas no-Prólogo, conl lo que no queremos decir que la influencia venga de estas escenas al Prólogo, sino simplemente que el poeta utiliza los mismos procedimientos para unas y para otras, puesto que en ambos casos se trata de comportamientos ya netamente teatrales.

III) Retardamicnto.-Una vez que ha tenido lugar la parte central de la escena y antes de que se produzca el período de acción, con el que dar paso a la escena siguiente, con cierta frecuencia Sófocles intercala un momento de detención en la marcha rectilinea de la acción, con ristas a intereses dramáticos extra-acción. Podemos establecer una división general primera en lo referente a su estructura. 
a) Esquema simple.-Cuando consta de una serie breve de elementos:

$$
\begin{aligned}
& \text { - d.e.3 + r.: E. C. } \\
& \text { - diálogo }\left\{\begin{array}{l}
\text { e.3 + d.e.3: Ay. } \\
\text { dim. an. +d.e.3: El. }
\end{array}\right.
\end{aligned}
$$

lo cual no aporta nada de interés, por cuanto que son esquemas estíquicos frecuentes en nuestro autor, y por lo tanto lo único interesante no es ya su estructura formal, sino la casi regularidad de un contenido y función tales en semejante distribución.

b) Esquema complejo.-Sin embargo en dos ocasiones, Ant. y Fil., no se trata de un breve período, sino que el poeta intercala todo un agón, aunque su función no es ya la tradicional en esta clase de escenas, sino que está en relación con este momento de retardamiento, y por lo tanto no se persigue ya que sea el vehículo de la obra. Pero hay una cosa que debe destacarse, y nos referimos al mantenimiento de la estructura formal de este tipo de contenidos, puesto que, aparte de otros elementos, el núcleo sigue siendo las dos resis enfrentadas seguidas del elemento dialógico tradicional, aunque, como es natural, está ausente 1a intervención del corifeo, puesto que el coro aún no ha llegado.

Resumiendo, de lo dicho en torno a este tercer período del Prólogo, el retardamiento, debemos sacar dos conclusiones generales: una, el empleo frecuente de este momento en una tal distribución, puesto que, de los ocho Prólogos analizados en Sófocles, cinco de ellos lo tienen; y dos, la tendencia a realizarlo preferentemente mediante un elemento dialógico, con vistas a una mayor viveza en la expresividad, to cual llega en algunos casos a convertirse en un auténtico agón.

IV) Acción.-Como es normal en la técnica dramática sofoclea, 1a escena del Prólogo, una más de las de corte estíquico ya teatralizadas, se cierra con un período de acción con el que la acción se lanza hacia adelante. En su estructura podemos trazar una más o menos precisa evolución, puesto que en las obras de época más antigua: $A y$., Traq., Ant. y E. R., encontramos un esquema más complejo-diversas intervenciones en resis- que en las tragedias posteriores: El., Fil. y E. C., donde suele aparecer un breve elemento, y concretamente en $E$. C. un corto diálogo entre padre e hija. Sin embargo, con vistas a un cuadro general de la técnica dramática de nuestro autor en este punto, apenas es interesante esta distinción, puesto que la variación es pequeña y sólo debe destacarse, si nos movemos en un plano de 
comportamiento general, el hecho de cómo Sófocles cierra la escena con un momento de acción expresado por un esquema de corte sencillo, compuesto por el emplco individual o repetido de breves resis, que en un caso es sustituido por un diálogo.

Alora bien, dentro de este período de acción debemos destacar el empleo dramático que hace Sófocles en un caso solamente, concretamente en $E$. $C$., de lo que podríamos llamar uacción interna». Nos estamos refiriendo a esa breve resis de acción que el poeta intercala entre el núcleo y el retardamiento, y que está orientada a hacer salir al §̧́vos del marco escénico, para dar paso precisamente al momento de retardamiento. Por lo tanto, se trata de un elemento de acción fuera de su distribución tradicional, desajuste éste motivado por razones de composición dramática.

En resumen, si pretendiéramos dar ahora una visión general de la estructura total del Prólogo en la obra de Sófocles, dejando a un lado los detalles particulares, deberíamos hacer notar cómo nuestro autor los compone con una gran regularidad dividiéndolos en cuatro partes básicas: una preparación, un núcleo, un retardamiento y un período de acción. Y cada uno de estos cuatro momentos suele tener una estructura que es frecuente en otras escenas igualmente estíquicas de corte teatral, pero que en el caso concreto del Prólogo contrasta con los esquemas simples de carácter antiguo de esta escena en estadios anteriores a nuestro autor. Por ello nos vemos empujados a establecer que en Sófocles, en 1o referente a la estructura, en su conjunto está ya bastante lejos de los tipos arcaicos, habiéndose convertido ya en una más o menos amplia escena estíquica. Ahora bien, también conviene precisar que se ha llegado a esta situación de complejidad por la fusión de una serie de elementos individuales que considerados en sí mismos tienen aún vestigios de arcaísmos.

\section{B) La distribución del Prólogo en Sófocles.}

Tras la estructura formal interna observemos lo que ya en otros trabajos anteriores hemos venido llamando distribución, es decir, la estructura formal externa.

En el punto primero de este trabajo explicábamos el sentido del análisis de la distribución en el caso concreto del Prólogo y Párodos, elementos éstos en contacto en los comienzos del Prólogo, dado el nacimiento de éste a partir del elemento inicial de la Párodos arcaica, aunque para más detalles enviamos allí, con lo que evitamos así una 
repetición innecesaria. Pues bien, en este sentido podemos establecer tres momentos ell la evolución dramática de Sófocles:

a) Pervivencia de la relación Prólogo-Párodos.-En Ay. y Traq. encontramos todavia un paralelismo de motives más o menos relajado entre Prólogo y Párodos. Lin $A n t$. los lazos entre ambas partes son ya aún menores, limitándose a un número reducido de alusiones, pero en líneas generales el centro del Prólogo en esta tragedia es algo ya separado de lo que el coro va a traer en la Párodos: en aquél Antígona expone la orden de Creonte y a la vez su plan de no obedecerle, mientras que el coro llega a la orchestra festejando el triunfo de su ejército frente al poder argivo, desconociendo lo que Antígona ha contado un momento antes; sin embargo todavía hay una serie de motivos comunes. Por todo ello, pensamos que esta tragedia supone el punto de transición a un segundo tipo que a continuación expondremos, y es por otra parte incierto dónde colocarla, si en este primer apartado o en el siguiente; si la hemos incluido en el primero ha sido fundamentalmente por razones de exposición, pero en realidad con igual legitimidad podría estar en el segundo; es en definitiva un escalón intermedio.

b) Falla de relación entre Prólogo y Párodos.-Un primer momento de innovación auténtica lo supone el hecho de que haya una ruptura total entre ambas partes iniciales de la obra. I esto, dejando a un lado 1a situación intermedia de Antigona, es lo que encontramos en Electra, donde el coro en su canto dialogado con Electra está muy lejos de los motivos del Prólogo.

c) Relación innovadora entre Prólogo y Párodos.-Sin embargo, el Prólogo, escena secundaria en sus comienzos, como ya hemos dicho repetidas veces antes, se consolida como una escena más de la obra, de tal manera que la acción va a dar verdadero comienzo en él, sin tener que esperar a la llegada del coro, como sucedía en el esquema arcaico, en un momento en que el coro era el componente básico y esencial del Teatro. En Fil. y E. C. notamos cómo la llegada del coro supone ya la realización del Prólogo, puesto que entra enterado de lo tratado en la escena estícjuica inicial de ambas tragedias, 1o cual supone desde una perspectiva de técnica dramática general, que el Prólogo ha ido consiguiendo una entidad plenamente independiente, y a partir de ahrora la relación entre ambas escenas no será la antigua de 1a Párodos hacia el Prólogo, sino la normal a lo largo de la obra, o sea, que una escena es continuación directa de la anterior, y en nuestro caso concreto del Prólogo hacia la Párodos. 
Ahora bien, esta nueva realidad está en cierta manera preparada por lo que hemos encontrado en $E$. $R$., donde el coro cn su plegaria, que en una gran medida repite, atnque ahora dirigida a los dioses, 1o que el sacerdote ha hecho con Edipo en la primera parte del Prólogo, es consciente también no obstante de la noticia traída por Creonte de Delfos en torno al mancillador que es necesario encontrar, notificación ésta que tiene lugar en la segunda parte del Prólogo.

En resumen, por lo referente a la distribución vemos en Sófocles una gradual, pero clara, tendencia innovadora a partir de situaciones más o menos antiguas, en este caso de la pervivencia de relación de motivos entre Prólogo y Párodos, a la implantación de una nueva situación, en la que el Prólogo ha adquirido rango de escena teatral independiente y funciona por sí mismo, lo cual por otra parte está en estrecho contacto con lo que acabamos de decir acerca de su estructura formal interna y de sus participantes, en donde veíamos una más progresiva consolidación de este paralelismo con las demás escenas estíquicas de dentro ya de la obra misma, es decir, a la otra parte de la Párodos.

\section{C) El contenido del Prólogo en Sófocles.}

En todos los Prólogos de nuestro autor observamos que el contenido central es la exposición de datos. Ahora bien, es necesario introducir una división dentro de este gran contenido narrativo, que nos aclare mejor la técnica dramática sofoclea:

a) Exposición de hechos pasados.-Es el valor auténtico del Prólogo en sus primeros estadios, cuando acaba de suplantar a los anapestos del corifeo que en un momento abrían la Párodos y la obra también. $\mathrm{Y}$ esto lo encontramos perfectamente en $A y$. y Traq., lo que nos vuelve a confirmar el carácter antiguo de estas tragedias. Uno o más actores de forma más o menos desarrollada exponían una serie de hechos previos a la acción de la obra, y a partir de ellos iba a dar comienzo ésta, ciendo su conocimiento necesario al auditorio para poder seguir de forma consciente desde el comienzo el desarrollo de la acción, lo cual en realidad, repetimos, no era ni más ni menos que lo que hacía el corifeo en sus anapestos pre-Párodcs, e incluso el coro mismo en la parte lírica de la Párodos, puesto que ya hemos dicho repetidas veces que incluso en elementos todavía rituales podían perfectamente intercalarse motivos narrativos.

b) Exposicion de hechos futuros.-Sin embargo, el contenido del Prólogo en Sófocles sufre una importante innovación, cuando vemos 
que ya no se narra una historia anterior a la acción de la obra que va a seguir a continuación, sino que descle estr. primer momento se programa en cierta medida los acontecimientos que van a tener lugar en la marcha misma de la obra. Es algo nuevo para la técnica teatral. Lo encontramos en El. y Fil., y lógicamente hay que ponerlo una vez más en relación con las diferentes innovaciones que ya hemos risto en otros niveles, y nos proporciona un dato más para la fijación del Prólogo, en las obras tardías de Sófocles, como una escena con entidad propia.

Ahora bien, entre un momento y otro podemos observar con claridad una etapa intermedia, en la que todavia es central la exposición de hechos pasados, pero también se alude en una mayor medida a la dirección que va a tomar la obra. $\mathrm{Y}$ este aparece ya perfectamente delineado desde Antígona, en donde el agón con que se cierra el Prólogo ya hace referencia a los posteriores enfrentamientos con Creonte, e igualmente en Edipo Rey con la notificación de Creonte de que el dios manda buscar al impuro, y también en Edipo en Colono y Rastreadores.

Eistos dos tipos generales con su zona de transición son los valores generales del contenido en el Prólogo de Sófocles, sin embargo al lado de ellos hay dentro de cada ejemplo en concreto otra serie de matices, que aquí no vamos a mencionar para no quitar claridad al esquema que pretendemos establecer, $\mathrm{y}$ a los que hemos hecho referencia en los análisis individuales que hemos renlizaclo en el punto anterior. $\mathrm{Y}$ así podríamos citar cómo la exposición de hechos pasados puede estar dividida en dos partes: una, los datos inmediatos a la acción que va a comenzar y que son los principales; y otra, que alude a unos hechos anteriores a estos últimos y que le sirven de preparación. De todas formas, to que debe quedar patente es el corte radical, en lo referente al contenido, que marcan esos dos tipos generales que hemos establecido.

\section{D) La función del Prólogo en Sófocles.}

Por lo que respecta a la función también encontramos en Sófocles dos etapas perfectamente distinguibles en su producción dramática, lo cual nos sirve a la vez para lograr una visión sincrónica y diacrónica de su obra:

a) Es base para la acción de la obra.-La exposición que el poeta busca con el Prólogo está orientada a enmarcar ante el auditorio la acción que va a comenzar con la llegada del coro a la Párodos. Y esto 
lo encontramos en $A y^{\prime}$. y Traq., lo que supone el mantenimiento en las primeras obras conservadas de nuestro autor del empleo originario de esta clase de escena, por cuanto que, como ya hemos visto, los anapestos del corifeo, a los cuales viene a sustituir esta escena estíquica, perseguían hacer mención del tema a causa del cual venía el coro ahora en su entrada en la orchestra, y cuando se produce la sustitución, el nuevo elemento hereda la función de su antecesor.

b) Planifica la acción de la obra.-Sin embargo, en la época tardía del poeta encontramos una alteración en la función tradicional, y así vemos cómo en El. y Fil. lo que se persigue es hacer un bosquejo de los puntos principales de la acción que va a seguir a continuación: Orestes y Odiseo planifican los diferentes pasos a seguir en sus respectivos cometidos, lo cual supone una clara innovación en la técnica del poeta. Y lógicamente, con esto se pierde el énfasis por la intriga en la marcha de la acción, característica ésta tan peculiar del hacer dramático de Sófocles en su primera época, y uno de cuyos reflejos más claros era el conocido estásimo "en falso", en que el coro tras una serie de peripecias inesperadas estalla en un canto de alegría, porque ve ya próxima la salvación de su Jefe $-\mathrm{y}$ el auditorio con él一. Sin embargo, con la composición de estos Prólcgos los espectadores al menos ya saben de antemano el camino por el que van a desarrollarse los acontecimientos, y por consiguiente el poeta elimina ese momento de "contraste fatal".

Pero igual que hemos visto en otros aspectos, también aquí en la función existe una zona de transición, en la que el valor antiguo de servir de base para que surja la acción se mantiene con plena vigencia, pero también conienza a vislumbrarse la nueva función mediante alusiones más o menos explicitas, es decir, tipos mixtos, como sucede con Ant., E. R., E. C. y Rastr. Is, en definitiva, un ejemplo más de la progresiva evolución a que se ven sometidos los diferentes aspectos y en resumidas cuentas toda la obra de nuestro autor, y lo que por otrat parte nos permite sacar una visión del plano diacrónico si queremos que el análisis sea más completo.

Ahora bien, hasta aquí hemos hecho alusión a las funciones que podrímos llamar principales, pero sin embargo también existen otras secundarias y particulares para cada obra ell concreto, y que consisten en matices más o menos intensos dentro de cada obra. Y así vemos 1a función de cfectivismo ciramático que se consigue en $A y$. y El. mediante la salida del héroc, en la primera obra, o el lamento de la desgraciada Electra, en la segunda. O tambiéin el matiz de ironía en el final de Edipo Rey, o en esta misma tragcdia la complicación que busca 
el poeta haciendo manifestar a Creonte que eran varios los que dieron muerte a Layo. De estos tipos semejantes cada ejemplo posee una serie de finalidades específicas para cada obra, a las que ya hemos ido haciendo alusión en sus análisis respectivos del punto anterior, y sólo los mencionamos aquí para dar constancia de su existencia.

Resumiendo, por lo tanto, en pocas líneas, en la medida de lo posible, este intento de sistenatización para conseguir un cuadro unitario del Prólogo en la obra de Sófocles, podemos decir que nuevamente nos encontramos con el hecho de que la estructura formal interna es la más innovada, habiendo llegado a un nivel importante de complicación de los esquemas antiguos, pero partiendo y estando compuesta de elementos antiguos, y sólo en una cierta medida alterados a su nivel inferior. Mientras que por el contrario, los aspectos del contenido y la función conservan una mayor regularidad al menos en la primera época de las obras conservadas, aunque también hemos de reparar en la tendencia evolutiva a que están sometidos.

\section{IV.-CONCLUSIÓN.}

Para terminar este trabajo dedicado a estudiar la estructura del Prólogo en Sófocles, conviene que a la luz de los datos expuestos en el punto inicial, acerca de la situación anterior a nuestro autor, y de los diferentes análisis realizados después en su producción dramática obtengamos finalmente un cuadro global, pero no de la técnica sofoclea considerada en sí misma, sino preferentemente ya en su relación con la realidad anterior a él, o sea, nos queda por afrontar el plano de la diacronía, pero dentro de la evolución general del Teatro, estudio éste que nos permitirá valorar mejor la aportación de nuestro autor al desarrollo del género dramático. En realidad ya hemos ido dando avances en lo dicho arriba, sin embargo, nos parece conveniente tratarlo ahora de forma reunida.

Si para empezar nos enfrentamos con el aspecto de la estructura formal deberemos hacer diversas consideraciones. En primer lugar hablemos de su realización en cuanto a la diversa participación de los actores. Decíamos al comienzo del trabajo que tal vez el esquema ori-

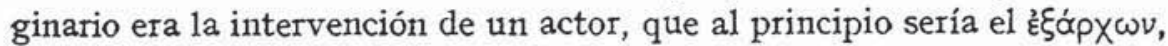

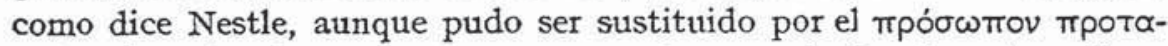
TIKóv; de todas formas un esquema a este respecto igualmente antiguo debió de ser la actuación simultánea de ambos, y más aún Esquilo

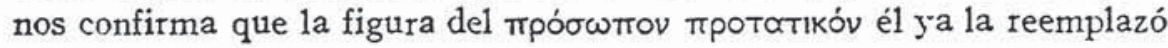


por un P.S. normal, es decir, con una posterior aparición en el interior de la obra misma. Pues bien, Sófocles utiliza todas estas realidades y las complica aún más. Veámoslo. De la actuación de un solo actor no conservamos ningún ejemplo suyo, sin embargo sí tenemos casos

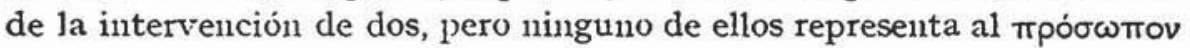

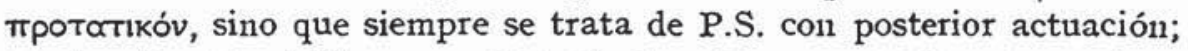

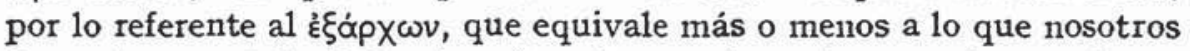
denominamos J.C., de los tres ejemplos con dos actores en dos ocasiones se mantiene este personaje-tipo, mientras que en una ocasión se ve reemplazado por el Op. Por lo tanto, respecto al esquema de dos actores encontramos aún en Sófocles pervivencias, aunque en todo caso también hay innovaciones. Ahora bien, la aportación mayor de nuestro autor en este sentido es la participación de tres actores en cinco de sus obras, y ya desde el mismo Ayante; de estos tres uno es siempre el J.C., y en algunos casos encontramos dentro de este esquema la presencia

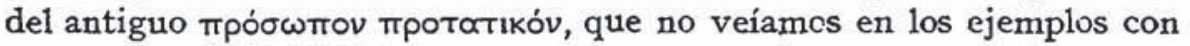
dos actores, pero de todas formas creemos que es interesante su presencia incluso en esta estructura más compleja, por cuanto que supone que todavía se mantiene en nuestro autor su vigencia, aunque él la. haya ya complicado con la intervención de un tercero. En resumen, en lo referente a la diferente participación de los actores notamos un cierto mantenimiento de restos antiguos, aunque existe ya un importante grado de innovación, debido al empleo de un tercer actor, con una gran frecuencia y además desde sus primeras obras conservadas.

Analicemos ahora el problema de su estructura formal interna. Al principio del trabajo decíamos que fundamentalmente basándonos en la obra de Esquilo podíamos rastrear que la estructura de los incipientes Prólogos debió de ser una resis, lo que sería sinultáneo con la participación del único actor. Al ampliarse el esquema a dos también experimentaría el mismo fenómeno la realidad formal y en ese caso tendríamos dos posibles resis con algún elemento dialógico, lo más probable tras ellas. Ahora bien, a partir ya de las últimas obras de Esquilo, y sobre todo con Sófocles, los esquemas simples se alteran en gran medida, y encontramos Prólogos con un importante índice de complejidad, como es lógico además con la aparición del tercer actor incluso en esta escena inicial. Pero veamos cómo se ha llegado a estos esquemas amplios. En realidad, se ha conseguido mediante el empleo de elementos inferiores. A decir verdad todos ellos han surgido por la confluencia proliferada de elementos inferiores de corte estíquico, es decir, a base de resis y partes dialógicas, ya d.e. ya $e$. Estas unidades elementales es evidente que son antiguas, pero sólo a primera vista, 
o mejor, una serie de ellas sí son arcaices, como las resis con función expositiva; sin embargo, hay otros componentes que, aún pareciéndolo por su forma y distribución, no lo son en realidad si tenemos presente su contenido y función con respecto a los demás elementos colindantes; y como ejemplo podriamos aludir a esas partes dialógicas, que abren una resis, en las que hemos notado el carácter de "anticipación", en lugar de ser simplemente un vehículo para que surja la resis. Pero hay más todavía, puesto que en algún caso observamos una casi total innovación, como son esos diálogos que funcionan de forma independiente, aunque éstos son pocos en números. Por lo tanto, debemos fijar un triple estadio en la clase de unidades elementales utilizadas para componer toda la escena: arcaismos, evoluciones e innovaciones.

Y será con el empleo frecuente de estas unidades con lo que el poeta llegará a componer los esquemas complejos que observamos. Ahora bien, si la estructura antigua, más simple, tenía que repartir los diferentes matices de su exposición en un número reducido de elementos formales, ahora no va a suceder lo mismo, y así vemos que cada indicación que el poeta quiere dar a entender utiliza toda la extensión que desea, y así hemos podido detectar cuatro partes casi constantes en todo Prólogo sofocleo: una preparación, un núcleo, un retardamiento y un período de acción-cierre de la escena. Para cada uno de estos momentos el poeta se sirve de la amplitud que le parece bien en aras de una completa expresividad, y así hemos ido viendo cómo Sófocles da una mayor o menor extensión a cada uno de esos cuatro períodos según los intereses específicos de cada obra en particular. En definitiva, Sófocles supone un estadio ya un tanto apartado de la época en que aún no se había logrado una importante desenvoltura escénica; él emplea el esquema de tres actores y esto le posibilita igualmente para incrementar la complejidad de la estructura en busca de una más rica expresividad; pero, eso sí, sirviéndose de elementos antiguos, aunque ya en una medida también innovados. $\mathrm{Y}$ todo esto se nos hace más patente si lo enfrentamos con gran parte de la obra de Esquilo, pero tal vez más aún si tenemos también en cuenta el período arcaizante de Eurípides, puesto que la obra de este último es más simultánea con la de Sófocles que Esquilo, y por ello resalta más la importante característica de compleja técnica dramática de nuestro autor.

Pero en realidad creemos que esta característica de los Prólogos sofocleos frente a los de los autores teatrales, es un reflejo más de la tendencia general a una mayor complejidad formal, to que significa para el presente trabajo que el poeta está utilizando en esta escena inicial de sus obras los mismos procedimientos que después aparecerán 
en el interior, en escenas igualmente estíquicas, y con esto no queremos decir que esté llevando su modo de hacer de dentro de la obra al Prólogo, sino simplemente que está empleando los mismos mecanismos en un sitio que en otro.

Pero hagamos mención también de otros aspectos no menos importantes. En cuanto a la distribución ya hemos visto cómo los primeros ejemplos conservados de su producción se mantienen todavía en una cierta medida de acuerdo con la situación anterior, aunque de todas formas suponen ya una serie de innovaciones parciales. Sin embargo, realmente a partir de Antígona ya observamos una clara tendencia a romper los esquenas arcaicos, lo cual se alcanzará plenamente en las obras tardías al introducir una nueva relación entre el Prólogo y la Párodos, lo que significará un replanteamiento de la naturaleza del Prólogo frente al resto de la obra y más concretamente frente a la Párodos.

En lo referente al contenido y a la función es donde encontramos un mayor conservadurismo. Hasta las obras de su última época aún se mantiene el valor originario del Prólogo como sucesor de los anapestos del corifeo, con que se abría la Párodos y la obra en definitiva en el estadio pre-Prólogo. Sin embargo, lógicamente se han perdido

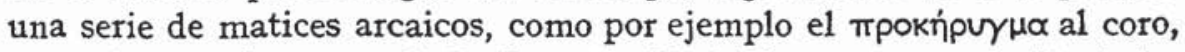
que aún en algunas resis-Prólogo de Eurípides se conserva. De todas formas, como decíamos hace un momento, con el desarrollo importante que adquiere la estructura formal en Sófocles, estos aspectos del contenido y la función se ven enriquecidos de rechazo, o tal vez mejor dicho, con la vista puesta en ellos es por lo que el pocta se debate por enriquecer el campo formal, aunque en realidad es el triunfo en este último el que determine el avance de los demás.

En resumen, el Prólogo en la producción dramática de Sófocles y dentro de la evolución general del Teatro, presenta una estructura formal interna y una distribución con un grado ya de importante innovación, sobre todo la primera, mientras que el contenido y la función mantienen un mayor conservadurismo, que de todas formas en su última etapa también se alteran. Por lo tanto, debemos una vez más afirmar que Sófocles supone para la evolución del Teatro el comienzo de una gran riqueza formal orientada a una mayor expresividad escénica, pero sin salirse de los valores fundamentales, que es la conclusión a que hemos llegado en estudios anteriores, y de la que lógicamente el Prólogo no es una excepción.

José María Lucas 